\title{
Bioinformatics and Cheminformatics Tools Applied to Chitosan and Derivatives with Biomedical Applicability
}

\author{
NATALIA GUMA ${ }^{1}$, ANDREEA NASTASE ${ }^{1}$, SPERANTA AVRAM ${ }^{*}$, RAMONA BUZATU ${ }^{2}$, \\ MARILENA MOTOC ${ }^{2}$ \\ ${ }^{1}$ University of Bucharest Department of Anatomy, Animal Physiology and Biophysics, Faculty of Biology, 36-46 \\ M. Kogalniceanu Blvd., 050107, Bucharest, Romania \\ ${ }^{2}$ Victor Babes University of Medicine and Pharmacy Timisoara, 2 Piata Eftimie Murgu, 300041, Timisoara, Romania
}

\begin{abstract}
Chitosan (CS) and its derivatives are important particles for the administration of nanomedicine and drugs. Using bioinformatics and cheminformatics tools, the molecular descriptors of chitosan and other new derivatives were quantified and compared using the nano-SAR method.
\end{abstract}

Keywords: chitosan, chitosan derivatives, molecular mechanism, bioinformatics, cheminformatics

\section{Introduction}

Chitosan presents two major properties such as degree of acetylation and molecular weight that affect its use as a matrix for drug delivery. These properties affect chitosan hydrophobicity and water solubility and, alter the efficiency of drug encapsulation. Chitosan is somewhat soluble in aqueous solvents. However, its solubility increases in an acidic environment, due to the protonation of amino groups. Chitosan presents a diversity of features that improve its usefulness as a drug delivery system. An important property of chitosan with implications in drug delivery is its mucoadhesivity and its ability to open epithelial junctions [1].

In the human body, chitosan is separated into amino acids and sugars, that are smoothly absorbed. Currently, CS and its derivatives are studied in many medical and pharmaceutical applications, including drug delivery, implants, bandages, tissue engineering and cellular encapsulation. CS contains several hydroxyl and amino functional groups that allow its bound to protein. It was mentioned that the chitosan with a degree of deacetylation of $50 \%$, is soluble in aqueous acidic medium. While CS is dissolved in an acidic environment, its $\mathrm{NH} 2$ groups are protonated, this allows CS strongly interacts with various molecules. It is suggested that this positive charge induces the CS antimicrobial activity during the interactions with the negative charged of cell membranes of the microorganisms [2].

Chitosan possesses amino and acetamido groups together with primary and secondary functional hydroxyl groups reactive at positions $\mathrm{C}-2, \mathrm{C}-3$, and C-6, respectively. The arrangement and distribution of these functional groups are the main factors that contribute to the structural, physical and chemical properties of chitosan [3].

It was reported that chitosan has shown little or no toxicity in animals and reduce adverse reactions have been reported in healthy volunteers [4].

Usually, low solubility of chitosan in biological solutions $(p \mathrm{H} \mathrm{7.4)}$ was reported, this reduces its application as a drug delivery vehicle [1]. Various methodologies have been proposed for CS solubilization, namely quaternation, alkylation, acetylation, carboxymethylation, conjugation of polyethylene oxide, chitosan formation/polyol salt combinations, generation of N-trimethyl chitosan [1].

Recently, pharmaceutical carriers represented by micelles, polymers, liposomes and nanoparticles are attractive targets. These systems presented a lot of advantages in improving the efficacy and safety of medicines. These systems can include both hydrophobic and hydrophilic active compounds, depending on the chemical structure of the carrier. They may also offer better stability for therapeutics against chemical and enzymatic degradation, longer drug influence on the target tissue, increase bioavailability, and drug targeting by including specific ligands.

*email: speranta.avram@gmail.com 
These systems are useful to transport small active molecules, peptides, proteins, vaccines and genes that are adsorbed, encapsulated, covalently and electrostatically attached to their surface [5].

Nanoparticles prepared with chitosan and its derivatives, frequently have a positive surface charge and mucoadhesive properties, so that they are able to adhere to the mucous membranes and release the useful drug load in a continuous release manner [4]. Chitosan-based nanoparticles presented various applications in drug delivery for cancer treatment, gastrointestinal disorders therapy, lung disease therapies or brain disorders therapy. Chitosan has reduced toxicity both in vitro and in vivo studies. The reduced toxicity of chitosan opens the new studies based on chitosan-based nanoparticles for nonparenteral drug delivery, chitosan features, toxicity, pharmacokinetics, and preclinical studies [4].

Chitosan is biodegradable and the process happens either by chemical or enzymatic catalysis [6]. Degradation of chitosan depends on the degree of deacetylation and the availability of amino groups. Also, chitosan is approved as safe by the US-FDA and EU for food and dressings. However, chitosan toxicity increases with increasing charge density and degree of deacetylation [7].

\section{Materials and methods}

\section{Computational strategy}

Molecular modeling and minimum energy calculation of natural compounds

In this study, we used 2D format of chitosan and its derivatives namely - chitosan malate, $\mathrm{N}$ acetylchitosan, chitosan-chloride, chitosan-silicate, heptyl-chitosan, N-(hydroxypropyl) chitosan, trimethylsilyl-chitosan, zinc-chitosan and chitosan-azide used SMILES (Simplified Molecular Input Line Entry System) files obtained from the PubChem database [8].

Table 1. The name of compound and pubchem database code

\begin{tabular}{|c|c|}
\hline Name of compound & PubChem CID \\
\hline Chitosan & 129662530 \\
\hline Chitosan malate & 3086339 \\
\hline N-acetylchitosan & 71306969 \\
\hline Chitosan-chloride & 129866077 \\
\hline Chitosan-silicate & 129840853 \\
\hline Heptyl-chitosan & 129847685 \\
\hline N-(hydroxypropyl) chitosan & 129682035 \\
\hline trimethylsilyl-chitosan & 129651913 \\
\hline zinc-chitosan & 129847607 \\
\hline chitosan-azide & 129855394 \\
\hline
\end{tabular}

The spatial structure (3D) of each compound was obtained used MOE10 (Molecular Operating Environment) software [9] and save in .mol2 file. After molecular modeling, the calculation of minimum potential energy was obtained using Forcefield MMFF94x at a 0.05 gradient. After energy minimization, Gasteiger partial charges were used.

\section{Calculation of molecular descriptors of chitosan and its derivatives}

To calculate the descriptors, we built the chitosan.mdb (MOE-specific) database, by applying the COMPUTE / QSAR mode and chose the descriptors offered by the software, in a number over 200. Considering our computational study on biomolecules [10], we calculated an important number of molecular descriptors belonging to (i) $2 \mathrm{D}$ descriptors including physical properties such as: steric features (subdivided solvent accessible surface and volume, subdivided van der Waals surface and volume), counts of atom and bond (hydrophobic and polar, rigid and rotatable bonds, hydrogen bond donor and acceptor atoms) and electronic descriptors (molar refractivity, dipole moment, molecular 
polarizability); and (ii) 3D molecular descriptors including potential energy descriptors, globularity, etc.

In the end, we selected a set of descriptors that were small enough to avoid redundancy and chance correlation, but large enough to allow an accurate validation of SAR study. The critical descriptors kept in our study were: the surface accessible to the solvent (ASA), solvent accessible surface calculated around polar atoms (ASA_P), Globularity (Glob), the partition coefficient of water/octanol $(\log \mathrm{P}(\mathrm{o} / \mathrm{w}))$, Solubility in water $(\log \mathrm{S})$, molecular flexibility $($ KierFlex) and frontier orbital energy LUMO.

Table 2. Definition of few molecular descriptors taken in consideration [8]

\begin{tabular}{|c|c|}
\hline Log P & $\begin{array}{c}\text { The ratio between a compound's concentration in a } \\
\text { given volume of n-octanol and concentration in a given } \\
\text { volume of water after octanol and water reached }\end{array}$ \\
\hline Glob & $\begin{array}{c}\text { Inverse condition number (smallest eigenvalue divided } \\
\text { by the largest eigenvalue) of the covariance matrix of } \\
\text { atomic coordinates. A value of 1 indicates a perfect } \\
\text { sphere while a value of 0 indicates a two- or one- } \\
\text { dimensional object. }\end{array}$ \\
\hline KierFlex & Kier molecular flexibility index: (KierA1) (KierA2)/n. \\
\hline ASA_P & $\begin{array}{c}\text { Water accessible surface area of all polar (|qi|>=0.2) } \\
\text { atoms. }\end{array}$ \\
\hline ASA & $\begin{array}{c}\text { Water accessible surface area calculated using a radius } \\
\text { of 1.4 A for the water molecule. }\end{array}$ \\
\hline Log S & $\begin{array}{c}\text { Log of the aqueous solubility (mol/L). This property is } \\
\text { calculated from an atom contribution linear atom type } \\
\text { model with } \mathrm{r} 2=0.90\end{array}$ \\
\hline Frontier orbital energy (LUMO). & $\begin{array}{c}\text { The energy (eV) of the Lowest Unoccupied Molecular } \\
\text { Orbital calculated by AM1 method }\end{array}$ \\
\hline
\end{tabular}

\section{Predicted molecular computational mechanism}

Trying to identify if these compounds have other molecular targets, we used Molinspiration bioinformatics tools [11] and SwissTargetPrediction [12]. Molinspiration software offers a wide range of chemoinformatics tools that support the handling and processing of molecules, including conversion SMILES and SDfile, normalization and fragmentation of molecules, calculation of different molecular properties required in QSAR, molecular modeling and drug design. We used Molinspiration /ModulePredicted bioactivity/. In this module, the bioactivity score [13] of molecules is evaluated at the active site of metabotropic receptors (GPCR), ion channel modulator, kinase inhibitor, nuclear receptor ligand, protease inhibitor, and enzyme inhibitor SwissTargetPrediction. This site allows predicting the targets of a small molecule. Using molecular similarities, compare the molecule with the 280'000 active compounds in the database with more than 2000 targets from 5 different organisms. The different output files that were obtained, contained: specific targets, probability of compounds to be a ligand for specific targets.

\section{Results and discussions}

\section{SAR analysis of chitosan and its derivatives}

Initially, all molecular descriptors in MOE/ QSAR descriptor module were calculated but, in the end, just a few of them were considered: ASA, ASA_P, Glob, $\log \mathrm{P}(\mathrm{o} / \mathrm{w}), \operatorname{logS}$, KierFlex (Table 2). Additionally, we calculated the energies of LUMO (eLUMO), which are very important in medicinal chemistry, and strongly recommended for analysis [14]. For avoided redundancy and chance correlation among the molecular descriptors, we selected a set of seven molecular descriptors that was large enough to allow an accurate validation of SAR analysis Here, the Pearson correlation matrix was evaluated. 
Table 3. Pearson correlation described the molecular features as independent variable.

\begin{tabular}{|c|c|c|c|c|c|c|c|}
\hline & ASA & ASA_P & glob & $\log \mathbf{P}(\mathbf{o} / \mathbf{w})$ & logS & KierFlex & AM1_LUMO \\
\hline ASA & 1 & & & & & & \\
\hline ASA_P & 0.47 & 1 & & & & & \\
\hline glob & -0.23 & 0.06 & 1 & & & & \\
\hline logP(o/w) & 0.19 & -0.40 & -0.19 & 1 & & & \\
\hline logS & -0.67 & -0.03 & 0.34 & -0.77 & 1 & & \\
\hline KierFlex & 0.60 & 0.35 & -0.36 & -0.02 & -0.28 & 1 & \\
\hline AM1_LUMO & 0.32 & -0.28 & 0.47 & 0.30 & -0.38 & -0.06 & 1 \\
\hline
\end{tabular}

Results of Pearson correlation presented in Table 3 show that the redundancy of molecular descriptors, as independent variables, was avoided (Table 3). This allows us to make a detailed analysis of similarities and differences of molecular descriptors described by the most active chitosan derivative as compared to chitosan.

The next step of our study was represented by the molecular features calculation and their spreading in follow clusters: (i) steric cluster represented by solvent accessible surface areas and solvent accessible surface areas around polar atoms; (ii) cluster represented by flexibility and globularity; (iii) hydrophobic cluster (expressed as $\log \mathrm{P}$ and account of hydrophobic atoms) represented by the capacity of compounds to cross the hydrophobic body barriers, (iv) the $\log S$ and electronic cluster represented by the values of frontier molecular orbitals energies (eLUMO).

In Table 4 we represented the values of molecular descriptors of chitosan and its derivatives.

Table 4. Predictive molecular descriptors of chitosan and its derivatives obtained in moe10 software

\begin{tabular}{|c|c|c|c|c|c|c|c|}
\hline Compound & $\begin{array}{l}\mathbf{A S A} \\
\left(\AA^{2}\right)\end{array}$ & $\underset{\left(\AA^{2}\right)}{\mathbf{A S A} \mathbf{P}}$ & glob & $\log P(0 / w)$ & $\log S$ & KierFlex & $\begin{array}{c}\text { eLUMO } \\
(\mathrm{eV})\end{array}$ \\
\hline Chitosan & 702.87 & 327.20 & 0.157 & -7.43 & 2.153 & 10.10 & 1.38 \\
\hline Chitosan malate & 923.44 & 461.49 & 0.062 & -8.046 & 1.165 & 16.47 & 0.35 \\
\hline Chitosan-azide & 711.34 & 320.61 & 0.066 & -7.564 & 2.365 & 10.23 & 0.14 \\
\hline Chitosan-chloride & 688.64 & 298.29 & 0.044 & -6.196 & 1.332 & 10.80 & 0.11 \\
\hline Chitosan-silicate & 721.23 & 392.22 & 0.090 & -6.386 & 1.888 & 10.27 & -0.79 \\
\hline Heptyl-chitosan & 880.94 & 299.45 & 0.042 & -4.199 & -0.951 & 13.69 & 1.65 \\
\hline $\begin{array}{c}\text { N-(hydroxypropyl) } \\
\text { Chitosan }\end{array}$ & 751.75 & 335.37 & 0.166 & -6.446 & 1.871 & 12.19 & 1.79 \\
\hline $\mathrm{N}$-acetylchitosan & 741.19 & 351.16 & 0.072 & -7.261 & 1.665 & 10.68 & 0.95 \\
\hline $\begin{array}{l}\text { Trimethylsilyl- } \\
\text { chitosan }\end{array}$ & 761.88 & 274.09 & 0.071 & -7.194 & 2.470 & 11.82 & 0.73 \\
\hline Zinc-chitosan & 695.80 & 321.49 & 0.047 & -7.116 & 2.293 & 16.07 & -0.38 \\
\hline
\end{tabular}

It is seen that all the studied molecules have $\log \mathrm{P}$ values between -8.04 and -4.19 . The low degree of lipophilia indicates that the molecules are likely to have good water solubility. Chitosan is not soluble under physiological conditions and therefore becomes soluble by functionalization.

$\log \mathrm{S}$ is an indicator of water solubility. Low solubility, in order, leads to low absorption and distribution characteristics. The commercial drugs generally have a $\log \mathrm{S}$ value greater than -4.00 . The $\log \mathrm{S}$ values of the studied compounds are between -0.95 and 2.47 , which indicates a good solubility.

The ASA values $\left(\AA^{2}\right)$ are between 274.09 and 461.49. The results show that chitosan malate and silicate have a higher solvent-exposed surface and therefore a higher reactivity compared to chitosan. In the case of $\log \mathrm{P}$ - the predictions show that heptyl chitosan, by functionalization can be a good transporter, far superior to chitosan.

Our flexibility calculations show that in the considered series, most compounds are similar to chitosan, except for chitosan malate and zinc-chitosan. This flexibility makes these carriers to be considered better than chitosan. 
In the case of globularity, $\mathrm{N}$ - (hydroxypropyl) chitosan and chitosan indicate the highest values, but still close to 0 , which suggests that they have the shape of a two-way or single object, while values close to 1 indicate the shape of a perfect sphere. Chitosan is used in the linear transport structure which needles are following our results. In general, our results show that these compounds are linear and can be used as a transport medium.

Very interesting results were obtained for eLUMO, energetic values vary from $1.79 \mathrm{eV}$ to $-0.79 \mathrm{eV}$, Heptyl-chitosan, N-(hydroxypropyl) Chitosan and chitosan having close values.

\section{Predicted molecular targets of chitosan and its derivatives in the body}

By applying the bioinformatics tools for chitosan and its derivates we predicted the possible molecular targets in the body. Molinspiration tools were used.

Table 5. The predicted molecular mechanism of chitosan and its derivatives

\begin{tabular}{|c|c|c|c|c|}
\hline Compounds & GPCR ligand & Ion channel modulator & $\begin{array}{c}\text { Protease } \\
\text { inhibitor }\end{array}$ & Enzyme inhibitor \\
\hline Chitosan & 0.04 & 0.02 & 0.36 & 0.41 \\
\hline Chitosan malate & -0.13 & -0.60 & 0.22 & 0.1 \\
\hline N-acetylchitosan & -0.1 & -0.17 & 0.38 & 0.36 \\
\hline Chitosan-chloride & 0.3 & -0.3 & 0.32 & 0.34 \\
\hline Chitosan-silicate & 0.14 & -0.01 & 0.39 & 0.50 \\
\hline Zinc-chitosan & 0.09 & 0.01 & 0.45 & 0.45 \\
\hline Chitosan-azide & 0.07 & 0.01 & 0.41 & 0.46 \\
\hline
\end{tabular}

From Table 5 it can be observed that chitosan and its derivatives presented a good probability (0.31-0.50) to inhibit the active site of enzymes, except chitosan malate (probability $=0.1$ ). All the chitosan derivatives mentioned in Table 5 have a good affinity at protease family active site (binding probability varies from 0.22 to 0.45 ).

To obtain more specific information about the molecular target of chitosan and its derivatives in the body, we applied the SwissTargetPrediction. The results of molecular targets, binding probability and common name of the receptor were presented in Table 6.

Table 6. Binding probability of chitosan and its derivatives in the body.

\begin{tabular}{|c|c|c|c|}
\hline \multirow{2}{*}{ Compounds } & Target & Name & Probability \\
\hline \multirow{2}{*}{ Chitosan } & & & $12 \%$ \\
\hline \multirow{2}{*}{ Chitosan malate } & Valinoid receptor & TRPV1 & $30 \%$ \\
\cline { 2 - 4 } & Muscleblind-like protein 1 & MBNL1 & $30 \%$ \\
\hline N-acetylchitosan & Muscleblind-like protein 2 (by homology) & MBNL2 & $70 \%$ \\
\cline { 2 - 4 } & & SELP & $70 \%$ \\
\hline Chitosan-chloride & P-selectin & SELE & $40 \%$ \\
\hline Zinc-chitosan & Microtubule-associated protein tau & MAPT & $30 \%$ \\
\cline { 2 - 4 } & Microtubule-associated protein tau & MAPT & $25 \%$ \\
\hline Chitosan-azide & Fibroblast growth factor & FGF1 & $30 \%$ \\
\cline { 2 - 4 } & Microtubule-associated protein tau & MAPT & $20 \%$ \\
\hline
\end{tabular}

After ruling the SwissTargetPrediction it was noticed that there is a diversity of proteins that can be considered as a target of chitosan ad its derivatives. We can mention that Microtubule-associated protein tau is a suitable target for Chitosan-chloride, Zinc-chitosan or Chitosan-azide while Nacetylchitosan strongly may inhibit selectins. 


\section{Conclusions}

Chitosan nanoparticles can efficiently deliver drugs to specific locations by retaining the drug locally to allow an extended time for drug absorption. The compounds studied by us so far have been little characterized in the literature, but quite often used in practice. Through this paper, we have succeeded in characterizing the cheminformatics and bioinformatics of the studied compounds. Important results we obtained from nano-SAR studies by identifying the most hydrophobic/ hydrophilic structures, those that can be used as an optimal delivery system.

Acknowledgment.This work was supported by a grant of the Romanian Ministry of Research and Innovation, CCCDIUEFISCDI, project number PN-III-P1-1.2-PCCDI2017-0728/2018, contract no 693/2018, within PNCDI III.

\section{References}

1.AHSAN, S., MATHAI, T., REDDY, K., SOORAPARAJU, S.G., ASTHANA, A., BHATNAGAR, I., International Journal of Biological Macromolecules, 110, 2017, p.97.

2.SHARIATINIA, Z., Advanced in Colloid and Interface Science, 263, 2019, p. 131.

3.YOUNES, I., RINAUDO, M., Marine Drugs, 13, 2015, p.1133.

4.MOHAMMED, M.A., SYEDA, J.T.M., WASAN, K.M., WASAN, E.K., Pharmaceutics, 9, 2017, p. 53.

5.VATANPARAST, M., SHARIATINIA, Z., Structural Chemistry, 29, 2018, p. 1427.

6.CRETEANU, A., STEFANACHE, A., VIERIU, M., TANTARU, G., OCHIUZ, L., Revista de Chimie, 70, 2019, p.1.

7.WANG, J.J., ZENG, Z.W., XIAO, R.Z., XIE, T., ZHOU, G.L., ZHAN, X.R., WANG, S.L., International Journal of Nanomedicine, 6, 2011, p.765.

8.***, https://pubchem.ncbi.nlm.nih.gov/

9.***, https://www.chemcomp.com

10.SEIMAN, D.D., BATALU, A., SEIMAN, C.D., CIOPEC, M., UDREA, A.M., MOTOC, M., NEGREA, A., AVRAM, S., Rev. Chim., 69, 2018, p.2377.

11.***, https://www.molinspiration.com

12.***, http://www.swisstargetprediction.ch/

13.***, https://www.molinspiration.com/docu/miscreen/druglikeness.html

14.REDDY, G.M.; GARCIA, J.R.; REDDY, V.H.; DE ANDRADE, A.M.; CAMILO A. JR.; PONTES RIBEIRO, R.A.; DE LAZARO, S.R., European Journal of Medicinal Chemistry, 123, 2016, p. 508.

$\overline{\text { Manuscript received: } 28.10 .2020}$ 